\title{
ELETROMIOGRAFIA DE SUPERFÍCIE DO MÚSCULO DIAFRAGMA EM PACIENTES SUBMETIDOS A TRANSPLANTE DE FÍGADO - DADOS PRELIMINARES
}

\author{
Surface electromyography of the diaphragm muscle in patients submitted to liver $t$ \\ ransplantation - preliminary data
}

\author{
Anna Claudia Sentanin 1 , Rayssa Pistilli Duarte', Aurea Maria Oliveira da Silva², Ligia dos Santos Rocetto Ratti', \\ Rodrigo Marques Tonella1, Ilka de Fátima Santana Ferreira Boin ${ }^{3}$
}

\section{RESUMO}

Introdução: Doença hepática crônica pode levar a alterações metabólicas e orgânicas que determinam a necessidade do transplante de fígado. O pós-operatório é um momento crítico para o paciente transplantado, principalmente em relação à avaliação e condução da hemodinâmica e da função ventilatória. Assim, a avaliação dessas alterações influencia a interrupção do suporte ventilatório e, consequentemente, a terapêutica respiratória desses pacientes. Objetivo: Avaliar a eletromiografia de superfície do músculo diafragma no pós-operatório de transplante hepático com e sem a utilização da pressão positiva. Métodos: Foram avaliados indivíduos de ambos os gêneros com idade entre 18 e 75 anos pós-transplante hepático, internados na UTI de adultos do Hospital de Clínicas da Unicamp. As informações do prontuário sobre os períodos pré, intra e pós-cirurgia foram anotados. O RMS (root means square) foi realizado através da eletromiografia do músculo diafragma após abertura da modalidade espontânea na ventilação mecânica e após extubação. Resultados: Foram selecionados sete indivíduos, sendo dois excluídos por não assinarem o TCLE, tendo composto o estudo cinco indivíduos: três do sexo masculino com idade média de $61 \pm 2,6$ anos. Não foram encontradas diferenças significativas entre o RMS da cúpula esquerda com e sem pressão positiva. Porém, houve diferença estatisticamente significante entre o RMS da cúpula direita com e sem pressão positiva $(p=0,043)$. Conclusão: Foi possível avaliar a eletromiografia de superfície do músculo diafragma no pós-operatório de transplante hepático. Verificou-se menor efetividade do músculo diafragma contra uma resistência, sem auxílio da pressão positiva, principalmente do lado do órgão transplantado.

Descritores: Unidade de Terapia Intensiva; Transplante de Fígado; Diafragma; Respiração Artificial; Extubação.

Instituições:

${ }^{1}$ Unidade de Terapia Intensiva, Hospital das Clínicas/Universidade Estadual de Campinas, Campinas/SP

2 Unidade de Transplante Hepático da Faculdade de Ciências Médicas - Universidade Estadual de Campinas, Campinas/SP

${ }^{3}$ Unidade de Transplantes de Fígado do Hospital de Clínicas da Universidade Estadual de Campinas, Campinas/SP

\section{Correspondência:}

Anna Claudia Sentanin

Rua Vital Brasil, 251, Cidade Universitária Zeferino Vaz,

CEP 13083-888, Campinas/SP

Tel: (19) 3521-8305 / 3521-7767.

E-mail: annasentanin@gmail.com

Recebido em: 07/11/2013

Aceito em: 19/12/2013

\section{INTRODUÇÃO}

Os pacientes com doenças hepáticas possuem diversas alterações orgânicas, metabólicas, dentre elas, a desnutrição, perda de massa e função muscular, gerando alterações respiratórias entre outros sintomas relacionados à hepatopatia que alteram significativamente a qualidade de vida desses pacientes. ${ }^{1,2}$

Existem diversos tratamentos existentes para as patologias hepáticas, destacando-se o transplante hepático, procedimento cirúrgico que pode causar lesão tecidual e muscular pela incisão realizada, pela manipulação das vísceras ou pelo uso de instrumentos afastadores que auxiliam na exposição do local a ser operado. ${ }^{3,4}$ A grande manipulação cirúrgica, lesão do nervo frênico durante o clampeamento da veia cava 
superior e ascite são as principais causas pós-operatórias para a diminuição da mobilidade diafragmática. ${ }^{5} \mathrm{~A}$ presença de ascite determina o aumento da pressão intrabdominal que é transmitida para o tórax, causando aumento da pressão pleural, elevação do diafragma e diminuição da mobilidade torácica. Isso resulta em padrão ventilatório restritivo e, consequentemente, aumento do trabalho respiratório. ${ }^{6}$

A eletromiografia de superfície é um método de estudo facilmente aplicável, não invasivo, por meio do qual são verificadas as magnitudes dos picos gráficos da atividade muscular voluntária ${ }^{7}$ e fornece informações eletrodiagnósticas importantes para a realização da pesquisa clínica. ${ }^{8}$

Sendo assim, o objetivo deste trabalho foi analisar os valores de RMS (raiz quadrática média) de ambas as cúpulas diafragmáticas através da eletromiografia de superfície do músculo diafragma no pós-operatório de transplante hepático e compará-los após o uso de ventilação mecânica invasiva com pressão positiva e após a extubação.

\section{MÉTODOS}

Trata-se de um estudo prospectivo e intervencionista, realizado no período de julho a dezembro de 2012, na Unidade de Terapia Intensiva (UTI) de adultos do Hospital de Clinicas da UNICAMP. O trabalho foi aprovado pelo Comitê de Ética em Pesquisa da Faculdade de Ciências Médicas da UNICAMP, sob parecer $n^{\circ}$ 922/2009, e todos os pacientes que aceitaram participar do estudo, assinaram o Termo de Consentimento Livre e Esclarecido.

\section{Critérios de Inclusão}

Foram selecionados indivíduos de ambos os gêneros, com idade entre 18 e 75 anos, pós-transplante hepático, internados na UTI de adultos do Hospital de Clinicas da UNICAMP, sem utilização de sedativos, hemodinamicamente estáveis, com quantidades mínimas de drogas vasoativas (Dobutamina $\leq 5 \mathrm{mcg} /$ $\mathrm{Kg} / \mathrm{min}$, Dopamina $\leq 10 \mathrm{mcg} / \mathrm{Kg} / \mathrm{min}$ e Noradrenalina de 0,05 a $0,1 \mathrm{mcg} / \mathrm{Kg} / \mathrm{min}$ ), que não apresentassem após a cirurgia sinais de sangramento ativo, com níveis normais de hemoglobina (acima de $8 \mathrm{~g} / \mathrm{dL}$ ) e em modalidade espontânea de ventilação mecânica.

\section{Critérios de exclusão}

Foram excluídos do estudo aqueles que necessitaram de ventilação mecânica não invasiva (VMNI) logo após a extubação ou os que se recusaram a participar da pesquisa e não assinaram o TCLE.

\section{Procedimento}

O procedimento consistiu inicialmente da coleta de informações através de uma ficha de avaliação que incluiu dados pessoais, valores de MELD (Model EndStage Liver Disease), hipótese diagnóstica e informações sobre a cirurgia. A eletromiografia de superfície foi realizada em dois momentos: primeiramente, com 0 indivíduo em modalidade espontânea, sob parâmetros mínimos, para que se prosseguisse a extubação (pressão de suporte $\leq$ a $10 \mathrm{cmH} 20$, Peep de $5-8 \mathrm{cmH} 20$, Fio2 $\leq$ $40 \%$ ), mantendo Glasgow $\geq 11$; repetida num segundo momento, logo após a extubação, aproximadamente 30 minutos, com suplementação de oxigênio para que se mantivesse a saturação periférica $\geq 90 \%$.

O paciente era posicionado em decúbito dorsal com $35^{\circ}$ de elevação da cabeceira e foi utilizado eletromiógrafo de superfície da EMG System do Brasil Ltda. série 00405 modelo 210C. Foram posicionados quatro eletrodos autoadesivos da marca $3 \mathrm{M}$ do Brasil e os locais de fixação destes foram previamente higienizados com álcool $70 \%$, sendo dois na região abaixo do osso xifóide e os outros dois a $16 \mathrm{~cm}$ de distância, no rebordo costal bilateral. ${ }^{9}$

A aquisição dos sinais foi feita em uma frequência de $500 \mathrm{~Hz}$ e a sensibilidade de amplitude do sinal de 500uV1, captado e processado por um software instalado em um notebook DELL IntelPentiun. Ao acessar o programa, foram visualizados os valores da atividade elétrica muscular obtida em um período de 10s determinado pelo software EMG System do Brasil, representado pelo RMS.

O programa utilizado para a análise estatística foi o SPSS versão 15.0 para Windows. Não foi encontrada distribuição normal das variáveis (teste KolmogorovSmirnov) e foi realizada a análise descritiva das variáveis numéricas e categóricas dos grupos. O teste de Wilcoxon foi aplicado para a comparação das variáveis relacionadas e o teste de Spearman para comparação das diferentes variáveis. Os valores de $p<0,05$ foram considerados como estatisticamente significantes.

\section{RESULTADOS}

Foram avaliados sete indivíduos, tendo sido excluídos dois, por não assinarem o Termo de Consentimento Livre e Esclarecido, totalizando cinco indivíduos. Desses, $60 \%$ eram homens e $40 \%$, mulheres. Os valores das características dos indivíduos do estudo estão na Tabela 1.

Em relação ao diagnóstico, três pacientes apresentavam cirrose hepática pelo vírus da hepatite C; um apresentava cirrose alcoólica e um, cirrose pós-biliar secundária. 
Anna Claudia Sentanin, Rayssa Pistilli Duarte1, Aurea Maria Oliveira da Silva, Ligia dos Santos Rocetto Ratti, Rodrigo Marques Tonella, Ilka de Fátima Santana Ferreira Boin

Os valores relacionados ao RMS de ambas as cúpulas diafragmáticas, bem como as diferenças entre eles, podem ser visualizados na Tabela 2 .

As diferenças entre os valores do RMS da cúpula esquerda com e sem pressão positiva não foram significativas $(p=0,225)$. Houve diferença estatisticamente significante entre o RMS da cúpula direita com e sem pressão positiva $(p=0,043)$

Não houve diferença significativa quando foram correlacionados os valores de MELD, sangramento e tempo cirúrgico com valores de RMS. Também não foram encontradas correlações significativas entre o T2 (tempo entre a abertura da modalidade espontânea e a extubação) e os valores de RMS.

Tabela 1 -Características da população com dados expressos em média e desvio padrão

\begin{tabular}{lc}
\hline \multicolumn{1}{c}{ Variáveis } & Valores \\
\hline Idade (anos) & $58,8 \pm 7,6$ \\
Altura (centímetros) & $168,2 \pm 11,36$ \\
Peso (quilogramas) & $76 \pm 9,5$ \\
MELD & $17,6 \pm 6,1$ \\
Tempo cirúrgico (minutos) & $438 \pm 12$ \\
Sangramento (mililitros) & $4000 \pm 361$ \\
T2 (minutos) & $192 \pm 113$ \\
\hline
\end{tabular}

T2: Tempo entre a abertura da modalidade espontânea e a extubação.

Tabela 2 -Valores do RMS com e sem a utilização de pressão positiva expressos em média e desvio padrão

\begin{tabular}{|l|c|c|c|}
\hline & RMS PP & RMS SPP & pvalor \\
\hline Cúpula direita & $31 \pm 12,64$ & $45,29 \pm 23,96$ & $0,043^{*}$ \\
\hline Cúpula esquerda & $32,13 \pm 9,34$ & $40,83 \pm 17,60$ & 0,225 \\
\hline
\end{tabular}

RMS: Root Means Square; PP: Pressão Positiva; SPP: Sem Pressão Positiva; *p valor: significância $\leq 0,05$

\section{DISCUSSÃO}

O sexo dos indivíduos submetidos ao transplante não apresentou nenhum impacto para a sobrevida do paciente, mas segundo Thuluvath e col., ${ }^{10}$ o sexo masculino apresenta maior incidência na realização do procedimento em comparação com o sexo feminino (2:1), o que corrobora com o perfil dos pacientes deste estudo, no qual $60 \%$ eram do gênero masculino. Barcelos e col. ${ }^{2}$ e Rocco e Soares ${ }^{11}$ demonstraram também incidência de $60 \%$ de indivíduos do sexo masculino para pacientes submetidos ao transplante hepático em um estudo clínico.

Os estudos de Silva e col. ${ }^{12}$ e Onaca et. al ${ }^{13}$ evidenciaram pior prognóstico em pacientes com MELD acima de 15. No presente estudo, a média dos valores de MELD pré-transplante foi de 17 e dos três pacientes que possuíam escores maiores, dois evoluíram com insucesso de desmame ventilatório e um com rejeição do órgão.

Dentre os pacientes analisados, a maioria apresentou diagnóstico de cirrose hepática $(\mathrm{CH})$ por vírus da hepatite C, da mesma forma que Barcelos e col. ${ }^{2}$ e Wertheime col. ${ }^{14}$ citaram em seus estudos.

O sangramento intraoperatório está associado ao aumento da morbidade e mortalidade pós-operatória. Durante o procedimento, o sangramento pode ocorrer como consequência da disfunção hemostática e da hipertensão portal em adição à própria cirurgia. ${ }^{15}$ Sankarankuttye e col. $^{16}$ avaliaram 41 pacientes submetidos a transplante hepático e observaram que 0 sangramento durante o procedimento cirúrgico foi em média de $10824 \pm 7002$ mililitros, sendo $270 \%$ a mais do que o encontrado no presente estudo, que foi em média de $4000 \pm 361$ mililitros.

Com relação ao tempo cirúrgico, foi observada média de $408 \pm 123$ minutos, sendo que dois pacientes com maiores tempos cirúrgicos apresentaram pior evolução e um deles foi a óbito. Isso vai de encontro ao estudo de Guerra e Lima ${ }^{17}$ que relacionaram o tempo cirúrgico de pacientes submetidos a ressecções hepáticas com a evolução para óbito entre o sétimo e o vigésimo-sexto dia de pós-operatório e constataram que pacientes que possuíam maior tempo cirúrgico tiveram evolução comprometida e foram a óbito.

Pacientes submetidos a transplante hepático sofrem influência do tempo de ventilação mecânica sobre a função e mecânica ventilatória, assim como descrito no trabalho de Rocco e Soares, ${ }^{11}$ que avaliaram 222 pacientes submetidos a vários tipos de cirurgia, sendo 147 transplantes hepáticos, que representaram o grupo com maior tempo de ventilação mecânica. Nesse contexto, Machado ${ }^{18}$ afirma que as cirurgias abdominais altas, por si só, levam à diminuição da força da musculatura respiratória já reduzida em pacientes pré-transplante, dificultando ainda mais o desmame ventilatório. Esses dados não estão de acordo com o presente estudo, já que neste, os pacientes foram extubados no pósoperatório imediato.

Silva e col. ${ }^{12}$ compararam a força muscular do diafragma entre indivíduos saudáveis e pacientes candidatos ao transplante hepático e observaram diminuição significativa da força muscular inspiratória nos 
hepatopatas. Além disso, demonstraram também que o RMS do músculo diafragma nos pacientes candidatos ao transplante $(56.56 \pm 34.64)$ foi maior do que o RMS de indivíduos saudáveis $(49.39 \pm 17.88)$, o que pode ser explicado pela adinamia e ascite encontradas em distúrbios hepáticos. No entanto, os valores observados nos cinco pacientes desta pesquisa foram inferiores aos valores do estudo de Silva e col., ${ }^{12}$ comparados com os indivíduos saudáveis e com os hepatopatas. Uma das possíveis justificativas para esses valores reduzidos do RMS é o fato de que quatro dos cinco pacientes permaneceram menos de um ano na fila de espera para o transplante.

Foi observado no estudo que o RMS da cúpula direita foi maior sem a pressão positiva, o que pode indicar piora da performance muscular diafragmática. Com a excursão diafragmática diminuída pós-transplante, a manipulação cirúrgica, principalmente do lado direito, pode justificar porque a função pulmonar torna-se deficitária, e, além disso, a distensão abdominal pós-transplante compromete ainda mais a função do músculo inspiratório. ${ }^{6}$ Além disso, notou-se que a pressão positiva auxilia a função muscular diafragmática, já que os melhores valores de RMS foram obtidos na presença desta.

Uma limitação importante deste estudo refere-se à amostra reduzida. Além disso, não foram avaliadas outras variáveis relacionadas à força muscular, bem como a evolução do paciente pós-alta da UTI e, portanto, novos estudos a respeito do tema são necessários.

\section{CONCLUSÃO}

Foi possível avaliar a eletromiografia de superfície do músculo diafragma no pós-operatório de transplante hepático. Verificou-se menor efetividade do músculo diafragma contra uma resistência, sem o auxílio da pressão positiva, principalmente do lado do órgão transplantado.

\section{ABSTRACT}

Introduction: Chronic liver disease can lead to metabolic and organic that determine the postoperative recovery of liver transplant. The post-operative period is critical to the transplanted patient, especially as to the assessment and conduction of the hemodynamic and the ventilatory function. Thus, the assessment of these changes influences the interruption of the ventilatory support, and consequently the ventilatory therapeutics for those patients. Purpose: To assess the perficial electromyography of the diaphragm in the post - liver transplantation with and without the use of positive pressure. Methods: Male and females patients with ages 18 to 75 years after liver transplantation in the $\mathrm{UCl}$ of the Unicamp Clinical Hospital. The pre, intra and post-surgery information were noted. The RMS (root means square) was performed by using electromyography of the diaphragm upon opening of the spontaneous mode on mechanical ventilation, and after extubation. Results: Seven individuals were selected, two were excluded as they did not sign the informed consent, and the study was comprised by five individuals, three males, mean age $61 \pm 2.6$ years. No significant differences were founded between RMS of the left dome with and without positive pressure. But there was a statistically significant difference between RMS of the right dome with and without positive pressure ( $p$ $=0.043$ ). Conclusion: It was possible to assess the superficial electromyography of the diaphragm in the post liver transplantation. It was verified an inefficient resistance of the diaphragm muscle without the assistance of the positive pressure, especially on the side of the transplanted organ.

Keywords: Intensive Care Unit, Liver Transplantation; Diaphragm; Respiration, Artificial; Airway Extubation. 
Anna Claudia Sentanin, Rayssa Pistilli Duarte1, Aurea Maria Oliveira da Silva, Ligia dos Santos Rocetto Ratti, Rodrigo Marques Tonella, Ilka de Fátima Santana Ferreira Boin

\section{REFERÊNCIAS}

1. Van Den Berg-Emons R, Van Ginneken B, Wijffels M et al. Fatigue is a major problem after liver transplantation. Liver Transplantation. 2006;12(6):928-33.

2. Barcelos S, Dias AS, Forgiarini Jr LA, Monteiro MB. Transplante hepático: repercussões na capacidade pulmonar, condição funcional e qualidade de vida. Arq. Gastroenterol. 2008;45(3):186-91.

3. Noujaim HM, Montero EFS, Ribeiro $C$ et al Estudo clínico prospectivo em humanos- comparando o papel da lesão de isquemia-reperfusão em enxertos esteatóticos VS não esteatóticos no transplante hepático. J Bras Transpl. 2010;13(1):1221-80.

4. Irwin S, Tecklin ST. Fisioterapia Cardiopulmonar, $3^{\circ}$ Ed. São Paulo: Manole,2003, p. 375-403.

5. Campo Silva TC, Carvalho R. Transplante hepático: problemas de enfermagem em pacientes no pós-operatório. J BrasTranspl.2006;(9):481-5.

6. Machado CS, Massarollo PCB, Carvalho EM et al. Efeito da força muscular respiratório pré-operatória no resultado do transplante de fígado. J Bras Transpl.2008; 11(3):948-53.

7. Oliveira AMS, Maturi S, Boin IE. Comparison of surface electromyography in respiratory muscles of healthy and liver disease patients: preliminary studies. Transplantation proceedings. 2011;43(4):1325-6.

8. González Roig JL, Lasoncel Herrera R. El laboratório de electromiografía em un centro de rehabilitacíon. Rev Cubana Ortop Traumatol.1996;(1):10.

9. Dionne A, Parkes A, Engler B et al. Determination of the best electrode position for recording of the diaphragm compound muscle action potential. Muscle\& Nerve.2009;(40):37-41.
10. Thuluvatha PJ, Guidinger MK, Fung JJ et al. Liver transplantation in the United States, 1999-2008. American Journal of Transplantation.2010;10(2):1003-19.

11. Rocco JR, Soares M. Prognóstico do paciente cirrótico admitido na terapia intensiva. Rev Bras Ter Intensiva.2010;22(1):11-8.

12. Silva AMO, Junior AC, Boin ISF. Profile of Respiratory Evaluation Through Surface Electromyography, Manovacuometry, and Espirometry in Candidates on the Liver Transplant Waiting List. Transplantation Proceedings.2012;44(8):2403-5.

13. Onaca NN, Levy MF, Sanchez EQ et al. A correlation between the pre-transplantation meld score and mortality in the first two years after liver transplantation. Liver Transplantation. 2003;9(2)117123.

14. Wertheim JA, Petrowsky H, Saab S et al. Major Challenges Limiting Liver Transplantation in the United States. Am J Transplant. 2011; 11(9):1773-84.

15. Cacciarelii TV, Keefe EB, Moore, DH et al. Effect of Intraoperative Blood Transfusion on Patient Outcome in Liver Transplantation. Arch Surg. 2009;13(4):25-9.

16. Sankarankutty AK, Teixeira AC, Souza FF et al. Impact of blood salvage during liver transplantation on reduction in transfusion requirements. Acta Cirurgia Brasileira. 2006;21(1):44-7.

17. Guerra EE, Lima LMP. Ressecções hepáticas com oclusão vascular aferente: analise de fatores de risco. Rev. Col. Bras.Cir. 2001;28(5):347-55.

18. Machado CS, Massarollo PCB, Carvalho EM et al. Efeito da força muscular respiratória pré-operatória no resultado do transplante de fígado. J BrasTranspl. 2008;11(3):948-53. 